\title{
Gas explosion in a room with a window and passage to an adjacent room
}

\author{
Yuri Polandov ${ }^{2}$, Aleksandr Korolchenko ${ }^{2 *}$ and Sergey Dobrikov ${ }^{1}$ \\ 1Orel State University, 302026, st. Komsomolskaya 95 Orel, Russia \\ ${ }^{2}$ Moscow State University of Civil Engineering, 129337, Yaroslavskoye Shosse 26, Moscow, Russia
}

\begin{abstract}
Some publications describe an effect, produced during a physical model experiment, when an adjacent gas-free room influences the gas explosion pressure in a room with a window. The explosion pressure in this case significantly exceeds ( 2.5 times) the explosion pressure in a room without an adjacent room. This result has been confirmed by our studies. Based on other available information about the influence of the ignition point location on the explosion pressure in one room, it was suggested that this could be true for an explosion in two rooms. In our studies we used a test unit with two connected chambers, each having a volume of $1.125 \mathrm{~m}^{3}$. It turned out that this influence of the adjacent volume was not so unambiguous as it was described in those publications. It was found out that the maximum effect of explosion pressure amplification by the adjacent room is achieved, when the igniter is located in the chamber filled with a gas-air mixture in the area between the center of the chamber and the window (maximum amplification by more than 3 times). This effect is lower directly by the window (1.8 times) and is practically absent in case of ignition within the area near the passage connecting the chamber with the adjacent room. This suggests that the effect discovered earlier is a special case of the general dependence of the gas explosion pressure in two chambers on the igniter location.
\end{abstract}

\section{Introduction}

Much attention has been paid to a gas explosion in a single room with a window. As judged by publications, the idea of the influence of an adjacent gas-free room, connected to the base room, on the gas explosion development was clearly shaped for the first time in works of researchers from the Moscow State University of Civil Engineering [1]. They showed that contrary to expectations the adjacent room serves not an explosion damper, but an explosion amplifier in case of an explosion in the base room. They conducted an experiment using two model chamber (base and adjacent): the base chamber was filled with a gas-air mixture, it had a window to the atmosphere and was connected with the adjacent chamber via a passage. It was found out that in case of ignition of the gas-air mixture in the center of the base chamber the maximum explosion pressure was considerably (2.5 times) higher in the presence of the adjacent chamber than that in a single chamber, not connected with an adjacent one.

\footnotetext{
${ }^{*}$ Corresponding author: KorolchenkoAYA@mgsu.ru
} 
As for the certainty of the gas explosion pressure amplification effect in a room connected with an adjacent room, where only the air is present, we have recently published materials [2] confirming the experiment results.

\section{Working hypothesis}

It is quite obvious that in case of a confined gas explosion the maximum explosion pressure does not depend on the ignition location. It is absolutely different in case of an unconfined gas explosion, for example, in a room with a window. It is known that the location of gasair mixture ignition in a room with a window affects the explosion process and the maximum pressure value [3-7]. According to these data, the farther is ignition location from the window, the higher is the maximum explosion pressure. This effects is more prominent, when the room length-to-width ratio is higher, and it can reach the multiplicity factor of more than 20. But in this case, the window location starts taking effect.

Presumably, in our case the gas ignition location will also affect the maximum pressure value of an explosion in a room with a window and a connected adjacent room. This statement is based on expected variants of the explosion development with different ignition locations. There are three variants:

First variant. The source is located near the passage to the adjacent room. In this case, mainly combustion products will get into the adjacent room rather than the gas-air mixture. There will be no combustion process in the passage or in the adjacent room, i.e. hot gases will just flow into the adjacent room. This room will serve a sort of damper.

Second variant. If the mixture is ignited near the center, it is expected that the unburned mixture will get into the adjacent room, where it will burn down. But in the passage the mixture will become turbulent, which will drastically increase the flame front area when it gets into the adjacent room resulting in the explosion pressure increase.

Third variant. The gas is ignited near the window. In this case, the largest amount of the mixture will get into the adjacent room, but the mentioned effect of the explosion pressure reduction, if the ignition location is near the window, will come into force. The explosion pressure will depend on the relationship between these two factors.

For higher credibility, experiments were conducted on a physical model.

\section{Research equipment}

In selection of a model unit we considered the influence of such factor as the room lengthto-width ratio on the gas explosion process. Keeping in mind the influence of another factor - the adjacent room - on the explosion development, we decided to minimize the influence of the first factor and selected a cubic shape of the both chambers. Photographs of the model unit are shown in Figure 1. It consists of two chambers No.1 and No.2 with the following dimensions: $0.5 \times 0.5 \times 0.5 \mathrm{~m}$ each. There was a disposal partition between the chambers, which separated the chambers in one case and connected them via a passage in the other case. Chamber No.1 had a vent window $0.15 \times 0.15 \mathrm{~m}$ (facing left in the figure). This window was provided with a door made of $1-\mathrm{mm}$ steel sheet and a damper position sensor. The igniter's positions could change between 5 points (numbered left to right). There is a fan inside the chamber to enable gas and air mixing and mixture preparation for ignition. The passage between the chambers is $0.15 \times 0.15 \mathrm{~m}$. While the mixture is being prepared, the passage is blocked from the side of the first chamber with a sheet of paper, which would be destroyed during the explosion. During simulation of explosions in a single chamber, a solid partition was installed between the chambers. Combustion products were 
removed from the chambers after explosions via the ventilation duct, which supplied the air to chamber No.2.

Propane from a cylinder was supplied to the first chamber. Experiments were conducted at concentrations near stoichiometric.

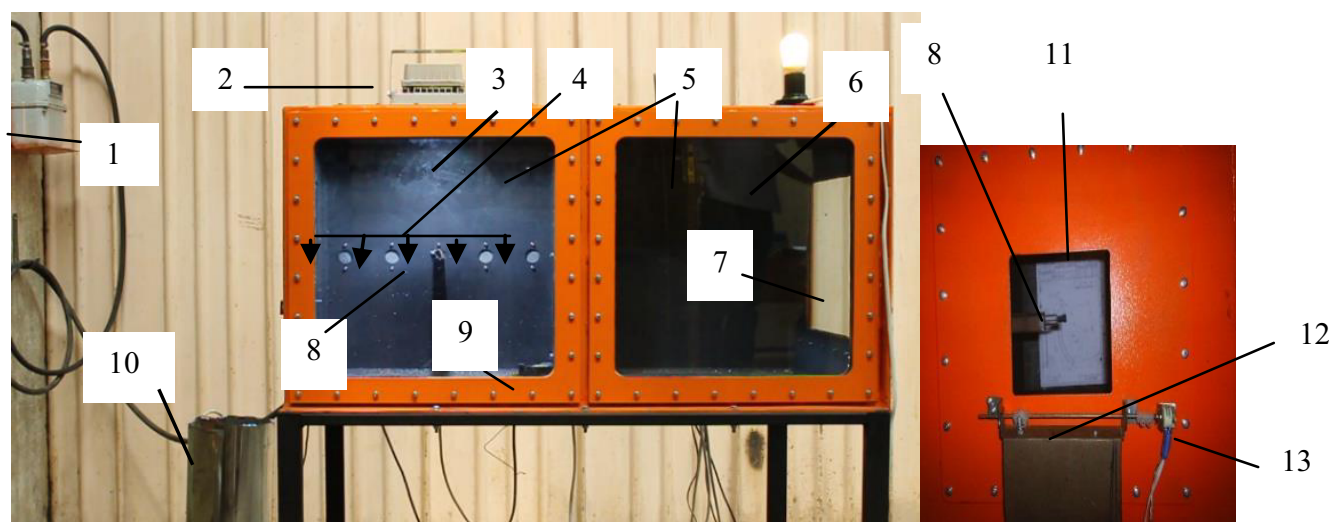

a) Side view of the unit

b) Front view of the unit

Fig. 1. General view of the unit: 1 - gas meter; 2 - backlight; 3 - chamber No.1; 4 -igniter locations (5 positions); 5 - pressure sensor locations; 6 - chamber No.2; 7 - back glass; 8 - igniter at position 3; 9 - circulation fan; 10 - ventilation duct; 11 - vent window; 12 - door (open); 13 damper position sensor.

\section{Standard tests}

Figures 2 and 3 show video snapshots of the gas explosion development in the single chamber and in two chambers respectively. It can be seen that in both cases, until the door opened, the flame shape was close to spherical, and after the door opened the flame front stretched in its direction. During the door opening the exiting jet is directed upward, deflected by the door. In Figure 2 the igniter is installed in position 3 and in Figure 3 - in the center of the chamber in position 3 . The explosion duration in the single chamber is noticeably shorter than in case of two chambers.
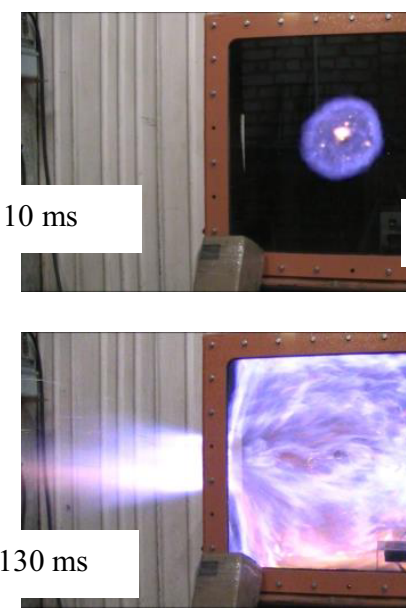
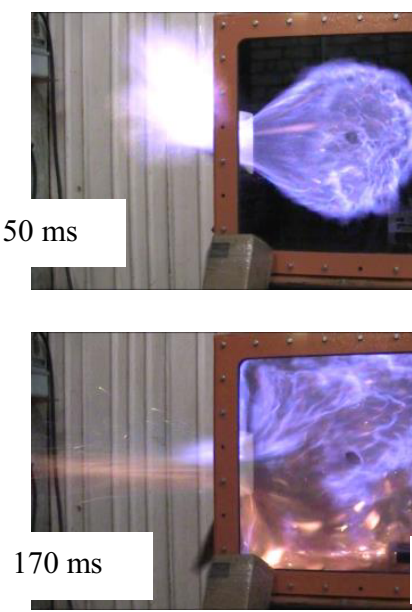
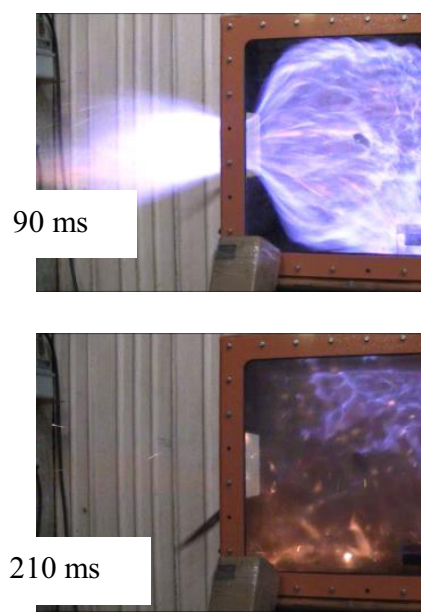

Fig.2. Video snapshots of an explosion in the single chamber. 

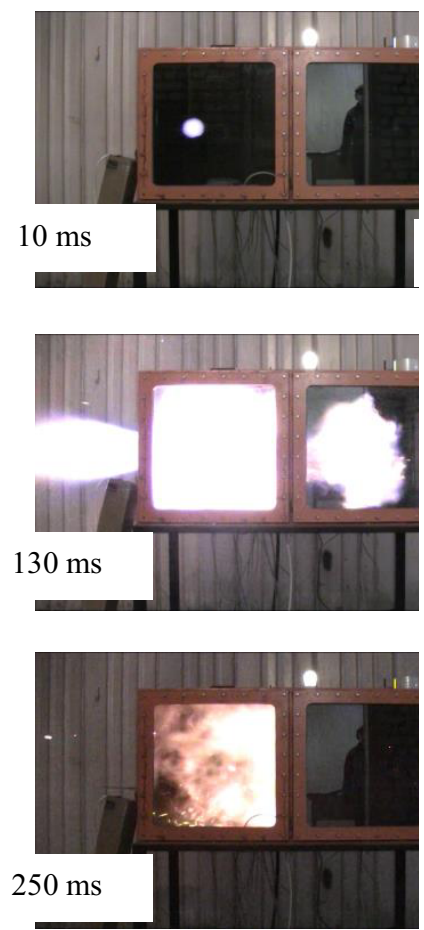
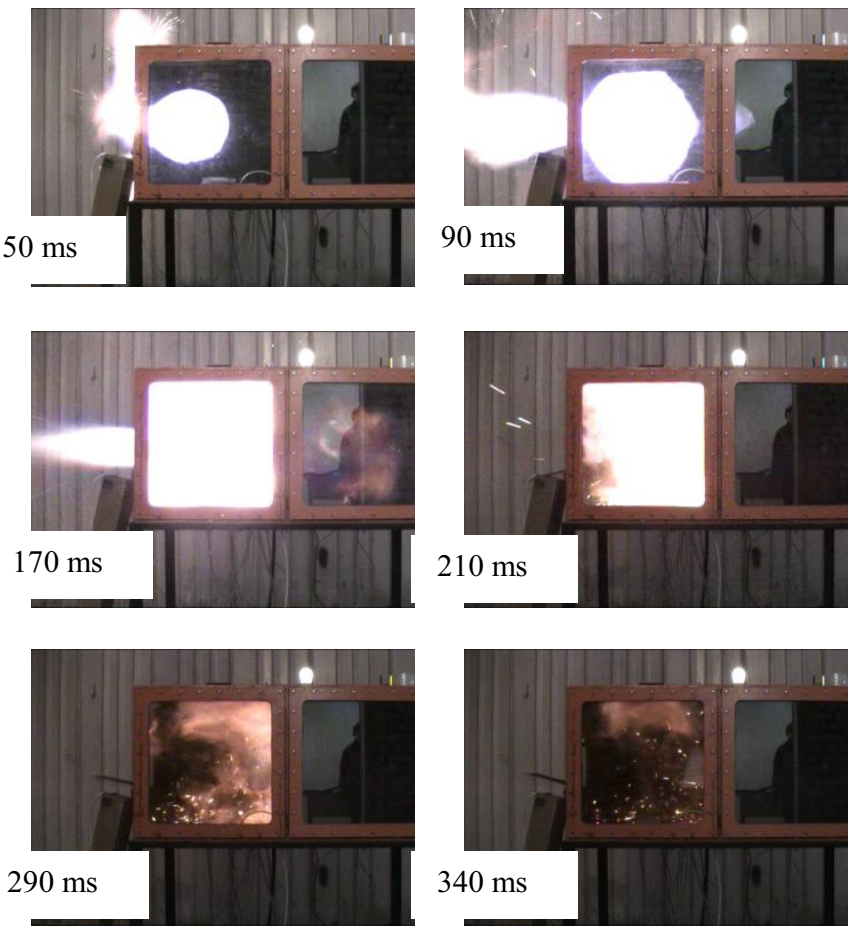

Fig. 3. Video snapshots of an explosion in two chambers.

\section{Data analysis}

The experiment program included two sets of tests followed by comparison of their results. The first set was conducted to study the dependence of the explosion development on the ignition location in the single chamber, and the second set included the same tests but with the open passage to the second chamber, blocked with a sheet of paper.

Tests with explosions in the single chamber. An explosion in a single chamber and snapshots of this process are not unique - such pictures are well known and we showed them for the sake of comparison with the explosion in two chambers and identification of differences. Test results of the first set with 5 positions of the igniter are summarized in a single diagram in Figure 4. Numbers of positions, at which explosions occurred, correspond to curve numbers. It can be seen that the closer the igniter to the window, the longer the explosion - in our case with reduction of the position number the explosion duration increases from 0.17 to 0.28 second.

Figure 6 shows a plot of maximum explosion pressure values in this set versus the igniter location (curve 2), whence it follows that while approaching the window the maximum pressure tends to reduction: in our case - from 0.1 barg to 0.006 barg. 


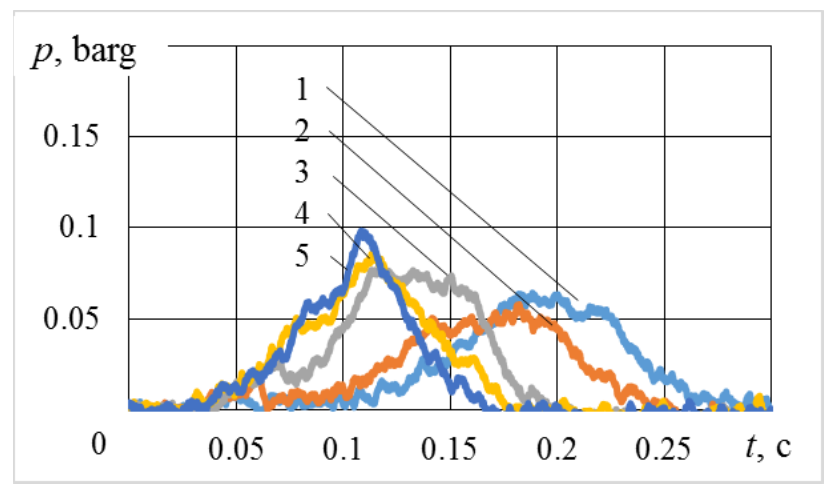

Fig. 4. Pressure trends for the single chamber explosion with different igniter's positions.

Tests with explosions in two chambers. Diagrams of the pressure development for explosions in two chambers at different igniter's positions are shown in Figure 5, and the dependence of the maximum pressures on the igniter's locations - in Figure 6. In this case, the obtained dependence of the explosion pressure against the igniter's location appears somewhat more complicated than in case of the single chamber explosion. When the igniter is placed near the passage to the second chamber (positions 4 and 5), the explosion pressure is even a little less than in case of the single chamber explosion. However, when the igniter is placed in the center of the chamber an near the vent window, the pressure is significantly higher than the single chamber explosion, especially in the second position. The following explanation of this phenomenon is possible. With the igniter installed near the passage, when the sheet is removed, already burnt gases get into the second room, i.e. these combustion products, an inert gas in this case, fill the volume of the second chamber. In this case, the second chamber serves as s damper.

When the igniter is moved deeper into the chamber, during the explosion the unburned mixture first enters the second chamber, becoming turbulent in the passage, and when it is ignited, it is burning across a developed area of the flame front. The further the igniter is moved from the passage, the more unburned mixture enters the second chamber.

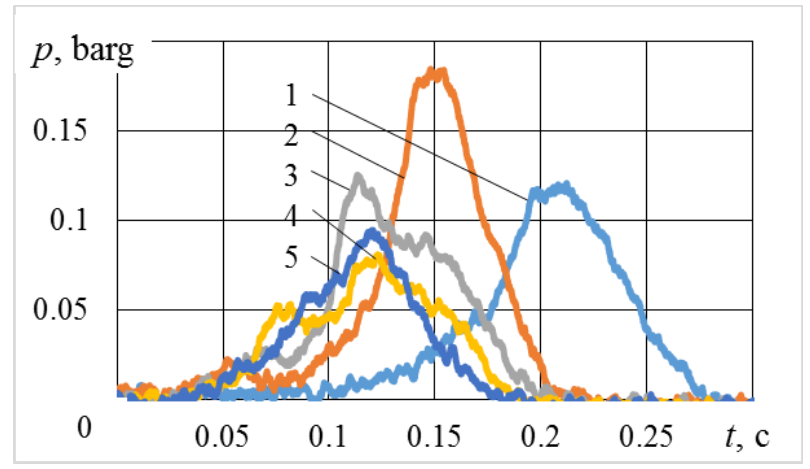

Fig. 5. Pressure trends for explosions in two chambers.

Finally, when the igniter is placed near the vent window, combustion products are discharged through the window, and they move with a high speed like high-temperature gases. Besides that, a part of the flame front is also released through the window into the atmosphere, which also contributes to explosion pressure reduction. 


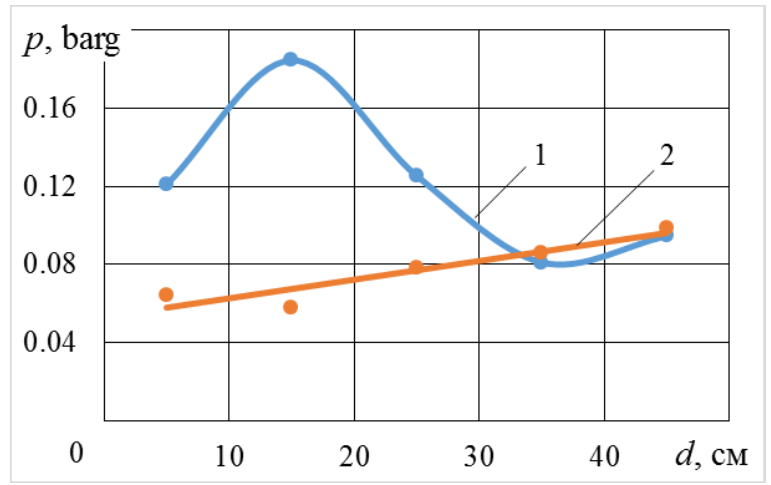

Fig.6. Dependence of maximum pressure values on the igniter's position 1 - with the adjacent chamber; 2 - for the single chamber.

To summarize the research results, we can review the dependence of the maximum explosion pressures on the igniter's location in two cases: first - in the single chamber, second - in the chamber connected with the adjacent chamber. In our case, the relative distance $L / L_{0}$ is in x-direction, where $L$ is the distance from the vent window to the ignition location; $L_{0}$ - the chamber length. The dependence $p_{2} / p_{1}=f\left(L / L_{0}\right)$ is shown in Figure 9. It is understood that the suggested dimensionless values do not fully meet the criteria, nonetheless they enable certain comparison of results obtained under different conditions.

Relation with earlier research results. It is interesting to refer to our earlier results [4], according to which a gas explosion in a single room with the ignition location at the farthest wall is much more dangerous than an explosion near the vent window. This refers to a single room with a window. It is clear that the data provided in this article contradict this statement and make controversial the selection of the gas stove location in a kitchen, which surely has an adjacent room, for example, a hallway.

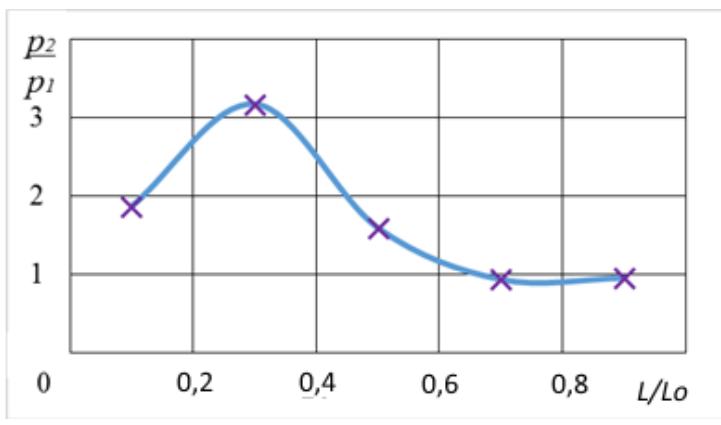

Fig.7. Comparison of maximum pressures of explosions with the adjacent chamber $\mathrm{p}_{2}$ and without it $\mathrm{p}_{1}$ at $\mathrm{L} / \mathrm{L}_{0}$.

\section{Summary}

It was found out that the previously discovered effect of the gas explosion pressure increase in a chamber with a vent window, when it is connected with a gas-free chamber, is highly influenced by the igniter's position in the first chamber. It turns out that this influence is not so unambiguous as stated in certain publications. Thus, with the gas is ignited near the passage to the adjacent chamber, the influence on the maximum explosion pressure is practically absent and even lower than in case of a single-room explosion. In this case, the 
adjacent room serves as an explosion damper. The influence manifests itself, as the igniter is mover to the center of the chamber and especially within the area between the center and the vent window, where it reaches a certain degree. If ignition occurs in close vicinity to the vent window, the maximum explosion pressure is less than in case of ignition near the center.

\section{References}

1. A.V. Mishuyev, V.V. Kazyonnov, A.A. Komarov, N.V. Gromov, A.V. Lukyanov, Fire and Gas Safety, 21 (2012)

2. Yu.Kh. Polandov, V.A. Babankov, S.A. Dobrikov, Fire and Gas Safety, 1 (2016)

3. Yu.Kh. Polandov, S.A. Vlasenko, M.A. Barg, Fire and Gas Safety, 3 (2007)

4. Yu.Kh. Polandov, V.A. Babankov, Zh. Fire and Gas Safety, 3 (2014)

5. C. R. Bauwens, J. Chaffee, S. Dorofeev, Combustion Science and Technology, 182(11) (2010)

6. G.G. Orlov, D.A. Korolchenko, A.Ya. Korolchenko, Fire and Explosion Safety, 24 (2015)

7. G.G.Orlov , D.A. Korolchenko, Fire and Explosion Safety, 24 (2015) 